\title{
Evolution of the Solvent Polarity in an Electrospray Plume
}

\author{
Rui Wang and Renato Zenobi \\ Department of Chemistry and Applied Biosciences, ETH Zurich, Zurich, Switzerland
}

\begin{abstract}
Solvent polarity plays an important role in electrospray ionization-mass spectrometry (ESIMS), one of the most widely used analytical methods for biochemistry. To have a comprehensive understanding of how solvent polarity affects ESI-MS measurements, we systematically investigated the polarity change in the ESI plume formed from an ethanol solution using laser-induced fluorescence (LIF) spectroscopy. Two solvatochromic dyes (i.e., dyes whose fluorescence emission is sensitive to solvent polarity), Nile red and DCM (4-dicyanomethylene-2methyl-6-p-dimethylaminostyryl-4H-pyran), were used as probes. The peak emission wavelengths of these two dyes exhibited significant red shifts $(8-12 \mathrm{~nm})$ when the measuring spot was moved away from the spray tip and in radial direction in the plume, indicating a dramatic polarity change during shrinking of the droplets. The emission intensities were also measured with a polarity-insensitive dye as a reference. The results are consistent with the peak wavelength measurements. Two key mechanisms responsible for the change of solvent polarity in the plume were considered, water entrainment from the surrounding air and solvent evaporation. Furthermore, quantitative analysis of the solvent polarity change was performed by using the Lippert-Mataga polarity parameter $\Delta f$. The value of $\Delta f$ reached 0.305-0.307 at the periphery of the ESI plume, which means that the solvent polarity in the smaller droplet is close to that of a mixture of $30 \%$ water and $70 \%$ ethanol $(\Delta f=0.307)$, even though the bulk solvent was ethanol containing less than $1 \%$ water as an impurity. (J Am Soc Mass Spectrom 2010, 21, 378-385) (c) 2010 American Society for Mass Spectrometry
\end{abstract}

$\mathrm{E}$ lectrospray ionization-mass spectrometry (ESIMS) is widely used in many important fields, e.g., biochemistry, the food industry, and pharmacy, and is still under fast development. Despite its huge success, only relatively few fundamental studies on the mechanism of ESI have been undertaken: the different modes of the electrospray [1, 2], partitioning effects in sprayed droplets [3-5], size, charge, and mass changes of the droplets during solvent evaporation [6-11], chemical equilibria in the electrospray process [12-14], and ion formation from the droplets [15-20] have been studied. However, more fundamental studies are still needed to make ESI-MS a totally reliable method for wider applications.

In this work, we focus on one fundamental aspect of the ESI process, the solvent polarity in the ESI plume. As one of the most basic physical properties, solvent polarity controls the rates of chemical reactions [21, 22] and chemical equilibria [23, 24] in solution, which have been widely studied by ESI-based techniques [25-27]. Especially, solvent polarity can have significant effects on biomolecules. For example, the charge state of biomolecules in ESI mass spectra can vary with solvent polarity $[28,29]$. The solvent polarity also influences

Address reprint requests to Professor R. Zenobi, Department of Chemistry and Applied Biosciences, ETH Honggerberg, HCl E 329, CH-8093 Zurich, Switzerland. E-mail: zenobi@org.chem.ethz.ch protein conformation [30], the stability of enzymes [31], and the structural characteristics of lipid bilayers [32]. Therefore, if the solvent polarity in the ESI plume changes, it will influence the chemical properties of analytes, and the final ion distribution in ESI mass spectra may no longer represent the analytes originally present in the bulk solution.

Despite its importance, there is very little literature concerned with the study of solvent polarity in electrospray plumes. In the interesting work by Zhou and Cook, the change of solvent composition in the electrospray plume was studied using a solvatochromic dye, Nile red $\left(\mathrm{C}_{20} \mathrm{H}_{18} \mathrm{~N}_{2} \mathrm{O}_{2}\right)$, in acetone/water and acetone/ ethylene glycol binary solvent mixtures using laserinduced fluorescence (LIF) [33]. They observed that the peak wavelength of Nile red exhibited a red shift along the spray axis and in radial direction, and attributed this phenomenon to the evaporation of the more volatile solvent component during the shrinking of the ESI droplets. The shift in the peak emission wavelength was used to derive the acetone content of the ESI spray plume at any given position. Their work indirectly indicates that the solvent polarity changes in the ESI plume.

Inspired by their work, we used two solvatochromic dyes, Nile red and DCM, to systematically and quantitatively study the solvent polarity in the ESI plume by LIF. The aim of our work was somewhat different than that in Zhou and Cook's publication: we mainly inves- 
tigated whether the solvent polarity change is the key reason causing variations in the fluorescence emission behavior in the ESI plume, and we investigated the possible mechanisms responsible for the solvent polarity change in detail. A single solvent, ethanol $(\mathrm{EtOH}$, frequently used for ESI-MS), was employed instead of solvent mixtures, to simplify the system. In addition to Nile red, another dye, DCM, was used to confirm the changes in solvent polarity. In Zhou and Cook's work, only the peak emission wavelength was measured, whereas in our work, the quantum yield was also evaluated to gauge the solvent polarity at different locations in the ESI plume, which was done with the aid of a reference dye. Other factors that could influence the fluorescence emission in the plume, temperature, and concentration, as well as partitioning effects, are addressed. Finally, the solvent polarity in the ESI plume was quantitatively analyzed using the Lippert-Mataga polarity parameter [34, 35], instead of Kosower's index [36], an empirical parameter used in [33], because the latter cannot be used to estimate the polarity of an unknown solvent composition without using a standard dye (1-ethyl-4-carbomethoxypyridinium iodide). Water entrainment from the surrounding air and solvent evaporation were found to be the most likely causes for the solvent polarity change.

\section{Experimental}

The ESI source and the LIF optical system are shown in Figure 1. The ESI source consists of a capillary (1715RNR; Hamilton, Bonaduz, Switzerland), a counter electrode and a high voltage supply (HCN140-12,500; FuG Elektronik, Rosenheim, Germany). The capillary is made of stainless steel. The length, i.d., and o.d. of the needle are $50 \mathrm{~mm}, 150 \mu \mathrm{m}$, and $720 \mu \mathrm{m}$, respectively. The diameter of the counter-electrode plate is $10 \mathrm{~cm}$.
The spray capillary, together with the counter electrode, is attached to a xyz stage to move the ESI assembly precisely with respect to the focus of the LIF optics. The sample was delivered by a $500 \mu \mathrm{L}$ syringe (1750 RNR, Hamilton) and a syringe pump (NE-1000; New Era Pump Systems, Wantagh, NY, USA). Different parameters of the ESI source were tested and it was found that it would operate stably in the stable cone-jet mode for a long time with a $20 \mathrm{~mm}$ capillary-plate distance, a supply voltage of $3.50 \mathrm{kV}$, and a flow rate of $5 \mu \mathrm{L} / \mathrm{min}$. We used these conditions in all the electrospray measurements. The current of the spray was monitored by measuring the voltage drop across the input resistance (1 M $\Omega$ ) of the oscilloscope (9350A; LeCroy, Chestnut Ridge, NY, USA) to ascertain the stability of the electrospray. It fluctuated by around $8 \%$ in the stable cone-jet mode, while fluctuations were around $20 \%$ in the pulsating Taylor-cone mode.

In the LIF optical system, the excitation laser $(\mathrm{Nd}$ : YAG, $532 \mathrm{~nm}, 40 \mathrm{~mW}$ ) is first expanded and collimated. Then the light is reflected by a dichroic mirror (Z532 RDC; AHF Analysentechnik, Tübingen, Germany) and focused by an achromatic doublet $(f=45 \mathrm{~mm}$, AC254050-A1-ML; Thorlabs, Newton, NJ, USA). The estimated beam waist of the focus spot is $\sim 10 \mu \mathrm{m}$ smaller than that in previous work $(\sim 1.3 \mathrm{~mm})$ in [33], and the estimated interrogation volume is only $\sim 3 \times 10^{-5} \mathrm{~mm}^{3}$. After that, the fluorescent signal is collected by the same achromatic doublet and focused onto the core of a multimode optical fiber (M25L05; Thorlabs), which guides the light into the spectrograph (Shamrock SR-163i; Andor Technology, Belfast, Ireland). Finally, the spectra are recorded by a Peltier-cooled chargecoupled device (CCD) camera (iDus DV420A-OE; Andor Technology).

Nile red, DCM, and rhodamine 6G (R6G) were obtained from Acros Organics (Geel, Belgium), with a

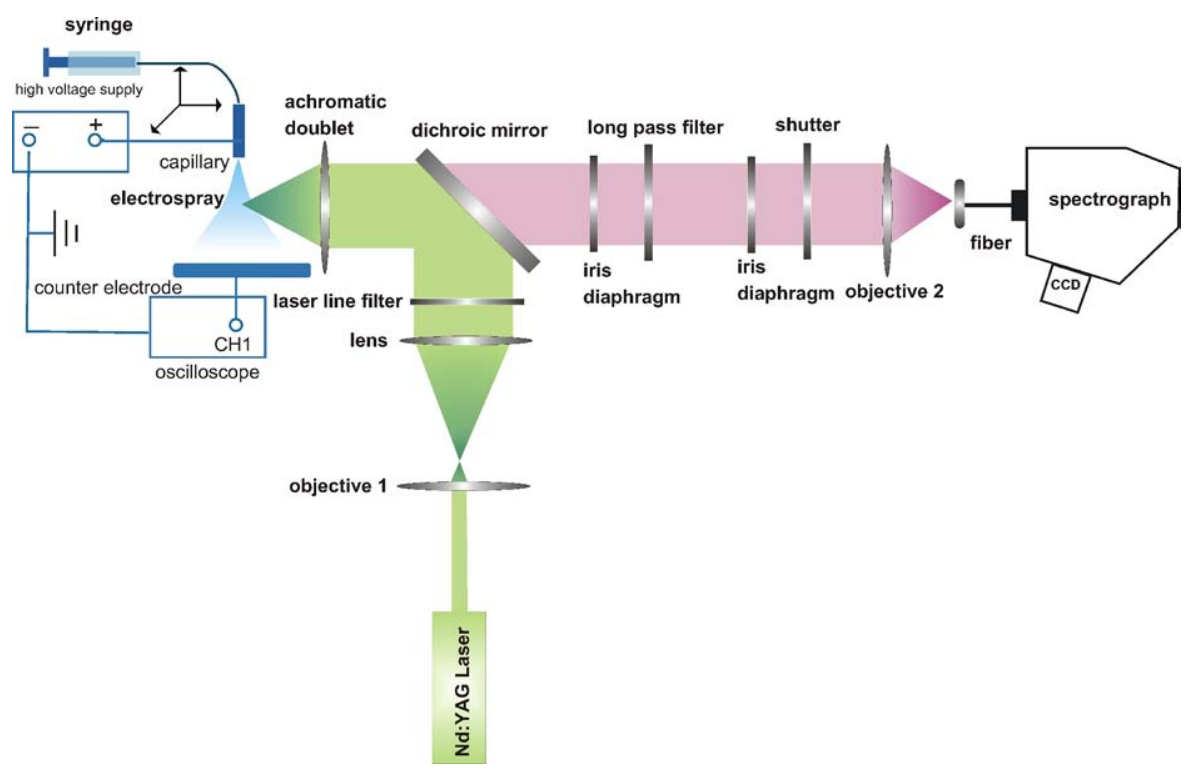

Figure 1. Set-up of the LIF measurement system. 
purity higher than $99 \%$. The peak wavelength of Nile red shows a red shift, and its quantum yield decreases when the solvent polarity increases because of a twisted intramolecular charge-transfer (TICT) excited-state [37]. For comparison, another polarity-sensitive dye, DCM, was used. It also exhibits a red shift of its peak wavelength with increasing polarity of the solvent, but its quantum yield changes in the opposite direction compared with that of Nile red [38]. To account for dilution effects, the quantum yield measurements of both Nile red and DCM were referenced against R6G, which shows no change in quantum yield in $\mathrm{EtOH}$ [39], according to the following equation [40]:

$$
\phi=\phi_{R} \times \frac{I n t}{I n t_{R}} \frac{A_{R}}{A} \frac{n^{2}}{n_{R}^{2}}
$$

where $\phi$ is the quantum yield of the sample, Int is the fluorescence intensity of the sample, $A$ is absorbance at the excitation wavelength, and $n$ is the refractive index of solvent. The subscript $R$ denotes the respective values of the reference substance. For the same solvent, $n$ should be equal to $n_{R}$. The quantum yield and peak wavelength of the R6G reference are neither influenced by aggregation in EtOH [39] nor by solvent polarity changes [41, 42]. Furthermore, the absorption spectra of dyes are generally much less sensitive to the solvent polarity than their emission spectra [43]. Therefore, the quantum yield of R6G, $\phi_{R}$, and the ratio of $A_{R} / A$ can be regarded as constants in the electrospray plume. The quantum yield $\phi$ is thus proportional to the intensity ratio, Int/Int ${ }_{R}$, in eq 1 . In the experiments, the intensity ratio was used as a measure to follow the quantum yields of Nile red and DCM change in the plume. The concentrations of Nile red and DCM in the solution used for the in-plume LIF studies are $15 \mu \mathrm{M}$. The fluorescence intensity ratio of these two dyes to R6G is close to 1 in the bulk solution.

The solvents used were $\mathrm{EtOH}$, water (analytical grade, $\geq 99.9 \%$ ) from Merck (Darmstadt, Germany) and acetonitrile (ACN, HPLC grade, $\geq 99.9 \%$ ) from SigmaAldrich (Steinheim, Germany). The water content in EtOH was measured by a Karl-Fischer Titrator (DL 38; Mettler-Toledo, Columbus, $\mathrm{OH}, \mathrm{USA}$ ).

All the fluorescence spectra of the bulk solutions were measured in a thin glass capillary $(0.3 \mathrm{~mm}$ diameter) to avoid self-absorption in solution, by the same LIF set-up, using an excitation wavelength of $532 \mathrm{~nm}$. The fluorescence spectra of the three dyes in bulk solution are shown in Figure 2. The peak emission wavelengths $\left(\lambda_{\text {max bulk }}\right)$ of Nile red, DCM, R6G in EtOH are $639.5 \mathrm{~nm}, 626.0 \mathrm{~nm}, 561.6 \mathrm{~nm}$, respectively, and that of Nile red in a mixture of $96 \%$ water and $4 \% \mathrm{EtOH}$ is $667.2 \mathrm{~nm}$, at a concentration of $15 \mu \mathrm{M}$. During the ESI plume experiments, the fluorescence spectra were mapped along the spray axis and in the radial direction ( $5 \mathrm{~mm}$ away from the ESI emitter) of the plume. A home-made program was used to separate the peaks,

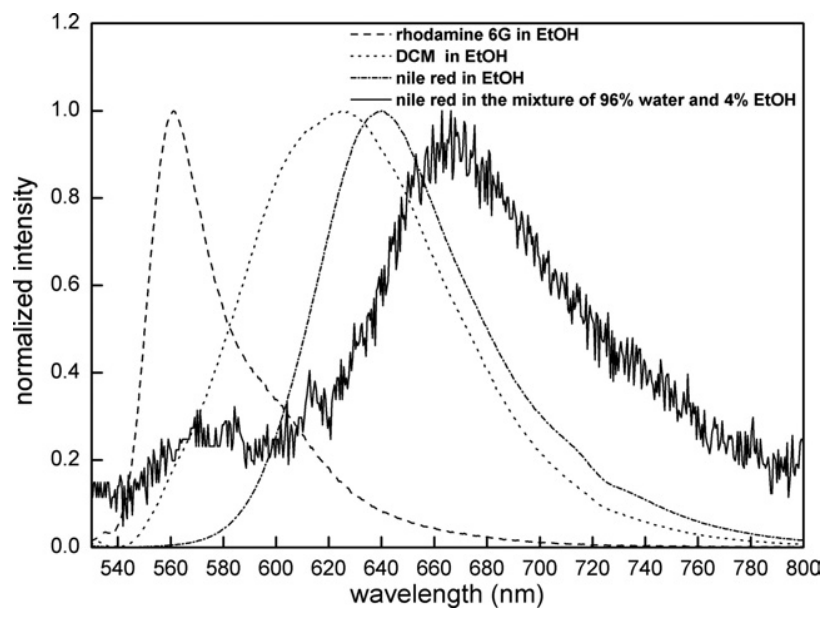

Figure 2. Fluorescence spectra of Nile red, DCM, R6G in EtOH, and Nile red in a solvent mixture of $96 \%$ water and $4 \% \mathrm{EtOH}$, with $15 \mu \mathrm{M}$ concentration.

extract the peak wavelengths, and evaluate the peak intensities of individual dyes.

\section{Results and Discussion}

\section{Spatially Resolved LIF in the ESI Plume}

Fluorescence measurements in the ESI plume were performed with different dye solutions (Nile red, DCM, and their mixtures with R6G), as shown in Figure 3. The peak emission wavelengths $\left(\lambda_{\max }\right)$ of Nile red and DCM in the plume are red shifted by $8-12 \mathrm{~nm}$ when moving away from the emitter on the spray axis by $15 \mathrm{~mm}$ (Figure 3a). In contrast, R6G does not show any wavelength shift. The trends and values of the peak wavelength shifts $\left(\Delta \lambda_{\max }=\lambda_{\max }-\lambda_{\max , \text { bulk }}\right)$ of Nile red and DCM are very similar no matter whether a single-dye or a two-dye solution was used. This means that R6G does not influence the fluorescence behavior of the other two dyes. The intensity ratios of Nile red and DCM to R6G in the spray direction are plotted in Figure $3 b$. The value of the intensity ratio of Nile red/R6G system decreased by more than $50 \%$, while that of DCM/R6G increased by $20 \%$ when the interrogation zone was moved by 15 $\mathrm{mm}$ away from the emitter. The quantum yields of Nile red and DCM are proportional to the intensity ratio according to eq 1 . Thus, clearly the quantum yield of Nile red decreases in the spray direction, while that of DCM increases.

The fluorescence behavior of these two dyes as a function of the radial position in the plume was also studied at a distance of $5 \mathrm{~mm}$ from the ESI spray tip. $\Delta \lambda_{\max }$ and intensity ratio changes in the two-dye system are depicted in Figure $3 c$ and d. The changes in $\lambda_{\max }$ for Nile red and DCM systems are very similar: it is small, $2-4 \mathrm{~nm}$, at the center of the plume (5 $\mathrm{mm}$ from the spray tip) and increases with distance from the center in radial direction. Moreover, the intensity ratio of Nile red to R6G decreases, while that of DCM to R6G increases 

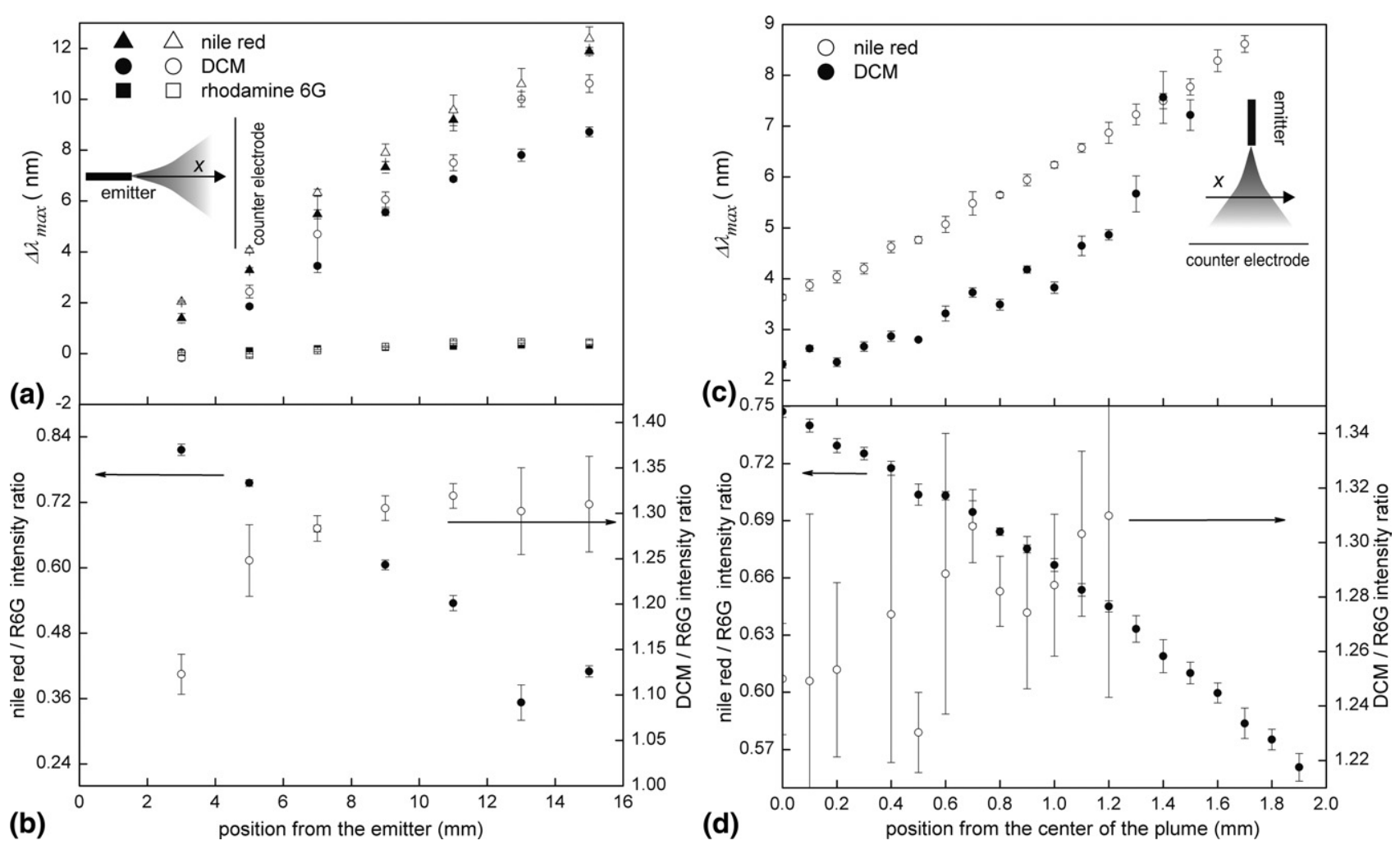

Figure 3. (a) $\Delta \lambda_{\max }$ of Nile red, DCM, and R6G in the single-dye system (open circles) and two-dye system (solid black dots). (b) The intensity ratios of Nile red/R6G and DCM/R6G; (a) and (b) are in the axial direction; (c) $\Delta \lambda_{\max }$ of Nile red and DCM in the two-dye system; (d) the intensity ratios of Nile red/R6G and DCM/R6G; (c) and (d) are in the radial direction, $5 \mathrm{~mm}$ away from the ESI emitter. The peak wavelength of the dyes in bulk solution $\left(\lambda_{\max , \text { bulk }}\right)$ : Nile red, $639.5 \mathrm{~nm}$; DCM, $626.0 \mathrm{~nm}$; R6G, 561.6 nm.

with radial distance. In the periphery of the plume or further away from the emitter, where smaller droplets prevail, the peak wavelengths of both two dyes have shifted significantly, and the quantum yield of Nile red decreased, while that of DCM increased. These observations imply that the solvent polarity increases significantly as the droplet diameters in the plume decrease.

Besides solvent polarity, other possible reasons that may cause changes in the fluorescence spectra of the dyes were also considered. Partitioning may cause smaller droplets at the periphery of the ESI plume to be enriched or depleted in some of the compounds, which can influence the measured intensity ratio. During the shrinking of ESI droplets, the dye concentration will increase, and the temperature of the droplet will decrease because of solvent evaporation. Aggregation should therefore be considered: some dyes aggregate when their concentrations increase and exhibit a redshift of their fluorescence emissions [44]. Another possible reason is the temperature. The fluorescence emission of thermochromic dyes is mainly sensitive to temperature. To evaluate the impact of the latter two factors, we measured the fluorescence spectra of Nile red in a cuvette at different concentrations (0.2-0.69 $\mathrm{mM} ; 0.69 \mathrm{mM}$ is close to the saturation concentration of Nile red in $\mathrm{EtOH})$ and temperatures $\left(-12^{\circ} \mathrm{C}\right.$ to $\left.72^{\circ} \mathrm{C}\right)$ in bulk solution (data not shown). Both a theoretical estimation, done by using the method described by Kebarle and Tang [18] and experimental results, obtained by phase Doppler anemometry [12] in the ESI plume indicate that the droplet size shrinks from 6 to $4.6 \mu \mathrm{m}$ when the measuring spot moves on the spray axis from 3 to $16 \mathrm{~mm}$ from the emitter. This means that the concentration only increases around three times due to droplet shrinking. Thus, the maximum concentration used, $0.69 \mathrm{mM}$, is above that expected in shrinking droplets for an initial concentration of $0.015 \mathrm{mM}$.

The temperature dependence shows that the peak wavelength of Nile red increases by less than $2 \mathrm{~nm}$ when the temperature drops from room temperature to $0{ }^{\circ} \mathrm{C}$. As far as the influence of concentration was concerned, the peak wavelength of Nile red was found to shift by less than $1 \mathrm{~nm}$ when the concentration increased 3000 times. Compared with the $8-12 \mathrm{~nm}$ peak wavelength shifts in the electrospray plume, the shifts caused by the temperature decrease and by possible aggregation of Nile red are much smaller. Thus, neither a temperature decrease nor aggregation can explain the observed changes of the fluorescence emission.

As proposed by Enke [3], partitioning of compounds can take place in an ESI droplet. After a Coulomb explosion, off-spring droplets preferentially incorporate 
material from the surface of the precursor droplets, i.e., if partitioning does take place, it can cause the chemical composition of the off-spring droplets to be different from that of the parent droplet. For example, Tang and Smith found that surface active compounds were enriched in the smaller droplets in an ESI plume [6]. In our experiments, the question is whether the intensity ratio of two dyes in the plume may be affected by partitioning effects. However, Nile red and rhodamine 6G have similar chemical structures, and both of them are not surface active, neither in $\mathrm{EtOH}$ nor in water. Any partitioning due to surface activity is thus expected to be very similar, and of minor importance. Even if a small fraction of the dye molecules reside preferentially at the droplet surface because they carry some of the charge, the intensity ratio should not be affected. We are thus focusing our attention on changes in the solvent polarity as the most reasonable explanation for the spectral change in the electrospray plume.

\section{Quantitative Analysis of the Solvent Polarity Changes in the ESI Plume}

To analyze the solvent polarity change in the plume quantitatively, the Lippert-Mataga polarity parameter $\Delta f$ was used. This parameter can be deduced by the following equation:

$$
\Delta f=\frac{\varepsilon-1}{2 \varepsilon+1}-\frac{n^{2}-1}{2 n^{2}+1}
$$

where $\varepsilon$ is the dielectric constant of the solvent and $n$ is the refractive index of the solvent. In eq 2 , the first term $(\varepsilon-1) /(2 \varepsilon+1)$ represents the low-frequency polarizability of the solvent and the second term $\left(n^{2}-1\right)$ / $\left(2 n^{2}+1\right)$ represents the high-frequency polarizability of the solvent. An equation can be used to describe the energy difference between the ground and excited states of dyes as a property of $\Delta f$, called the LippertMataga equation [34, 35],

$$
\Delta v=v_{A}-v_{F}=\frac{\lambda_{F}-\lambda_{A}}{\lambda_{A} \times \lambda_{F}}=\frac{2}{h c} \Delta f \frac{\left(\mu_{E}-\mu_{G}\right)^{2}}{a^{3}}+\text { constant }
$$

where $\lambda_{A}$ and $\lambda_{F}$ are the wavelengths of the absorption and emission maxima of the dye, respectively, $h$ is Planck's constant and $c$ is the speed of the light; $\mu_{E}$ and $\mu_{G}$ are the dipole moments of the excited state and ground state of the dye; $a$ is the radius of the cavity in which the dye resides. Since the absorption spectra of dyes are generally much less sensitive to the solvent polarity than their emission spectra [43], the value of $\lambda_{A}$ can be considered roughly as the constant, $(550 \mathrm{~nm}$ in $\mathrm{EtOH}$ [45]) to simplify the estimation of the solvent polarity in the plume. The values of the slope, $2\left(\mu_{E}-\mu_{G}\right)^{2} / h c a^{3}$ and the intercept were obtained by fitting the spectral results of Nile red in a series of water/EtOH mixtures of the bulk solution by eq 3 . The dielectric constant $\varepsilon_{M S}$ and refractive index $n_{M S}$ of a water/EtOH mixture can be calculated by the following equations [46]:

$$
\begin{aligned}
& \varepsilon_{M S}=f_{W} \varepsilon_{W}+f_{E} \varepsilon_{E} \\
& n_{M S}^{2}=f_{W} n_{W}^{2}+f_{E} n_{E}^{2}
\end{aligned}
$$

where the subscripts $\mathrm{W}$ and $\mathrm{E}$ represent water and $\mathrm{EtOH}$, respectively, and $f$ represents their volume fractions. Dielectric constants $\varepsilon$ and refractive indices $n$ of pure water and $\mathrm{EtOH}$ are 78.3, 24.3, and 1.33, 1.35, at $20{ }^{\circ} \mathrm{C}$ respectively $[43,44]$. From this, the LippertMataga parameter $\Delta f$ of a water/EtOH mixture can be obtained from eq 2 . The experimental data and the Lippert-Mataga plot calculated by a linear fit are shown in Figure 4a. Clearly, the experimental data exhibit a good linear relationship between $\Delta f$ and $\Delta v\left(R^{2}=\right.$ 0.9863). The value of $\frac{2\left(\mu_{E}-\mu_{G}\right)^{2}}{h c a^{3}}$ is 22.49 and $\Delta f$ in the ESI plume can then be calculated from eq 3 as shown in
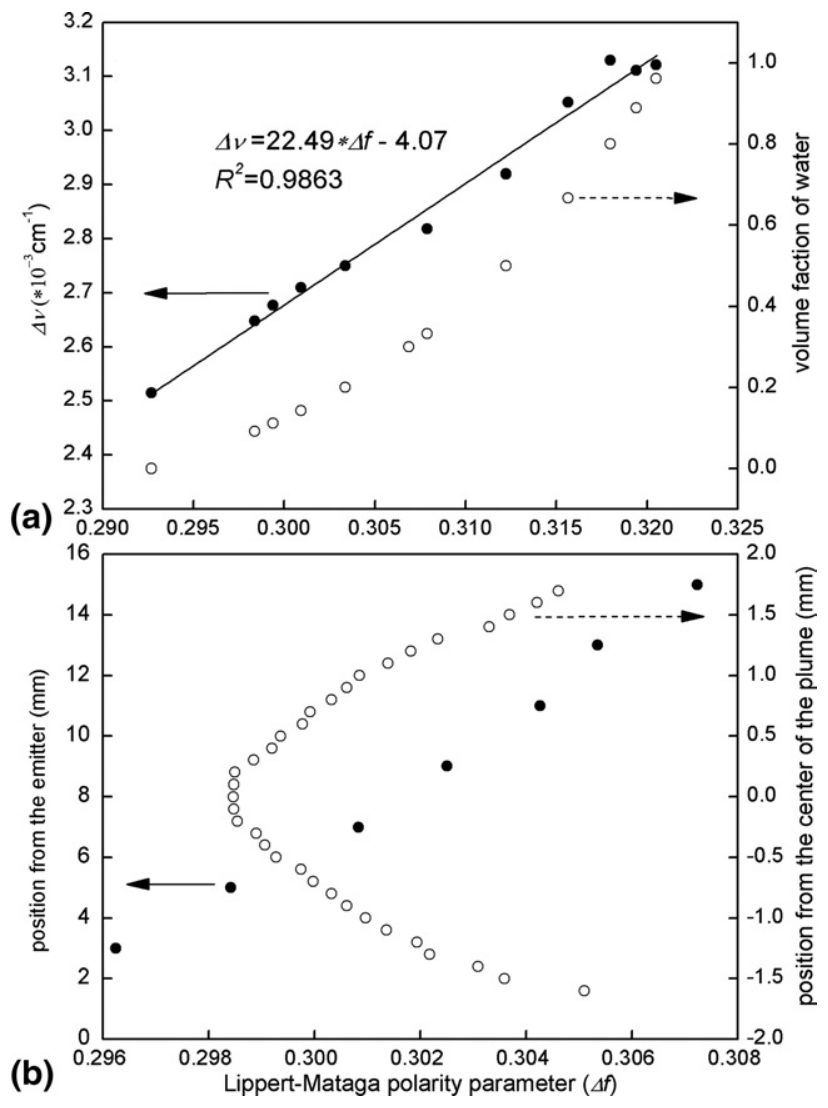

Figure 4. (a) Experimental data ( $\Delta v$ as solid black dots: volume fraction of water as open circles) and linear-fitting plot (line) of Nile red in various compositions of water and EtOH mixtures. (b) The values of $\Delta f$ in spray (solid black dots) and radial (open circles) directions in the electrospray plume. 
Figure $4 \mathrm{~b}$. The value of $\Delta f$ increases from 0.296 near the spray needle to 0.305 at the periphery of the plume and to 0.307 at a vertical distance of $15 \mathrm{~mm}$ from the ESI emitter. For pure $\mathrm{EtOH}$ and water, the values of $\Delta f$ are 0.293 and 0.321 , respectively. This means that the solvent polarity in smaller droplets is close to the polarity of a mixture of $30 \%$ water and $70 \%$ ethanol $(\Delta f=0.307)$, even though the bulk solvent is ethanol containing less than $1 \%$ water as an impurity.

\section{Mechanism of the Solvent Polarity Change}

The above discussion shows that a larger solvent polarity is associated with the shrinking of the electrospray droplets. The most obvious explanation for this could simply be solvent evaporation: $\mathrm{EtOH}$ evaporates faster than water because of its higher vapor pressure. This is also true for only very small amounts of water, because a mixture of water and ethanol will not form an azeotrope when the temperature is below $305.7 \mathrm{~K}$ [47]. The percentage of water, initially present at trace amounts, will increase in the droplet when less EtOH molecules remain, due to the faster evaporation of the latter, resulting in a solvent polarity increase as the ESI droplets shrink. Thus the dependence of the peak wavelength of Nile red in EtOH as a function of initial water content was measured along the spray axis. A wide range of water contents between $0.02 \%$ and $46.78 \%$ was chosen. The results are shown in Figure 5a. Surprisingly, the shift of $\mathrm{EtOH}$ with $46.78 \%$ water content is still close to $7 \mathrm{~nm}$, even a bit higher than for EtOH with $0.43 \%$ water. If solvent evaporation were the only mechanism responsible for the solvent polarity change, the shift of Nile red in $\mathrm{EtOH}$ with $46.78 \%$ water content would be much less than that of Nile red in EtOH with $0.43 \%$ water content. This indicates that solvent evaporation cannot be the only mechanism causing the change in solvent polarity.

For EtOH with a very low water content, water entrainment from the surrounding air may contribute. Several studies have already shown this: Innocenzi and coworkers studied the evaporation of $\mathrm{EtOH}$ and water from droplets by time-resolved infrared spectroscopy and found that water entrainment from the atmosphere occurred when the water content in the solution was less than 1\% [48]. Katta and Chait observed water entrainment into ESI droplets in a study on H/D exchange [49]. Zhou and Cook sprayed a nonaqueous acetone/ethylene glycol sample without nebulizing gas [33]. They also found that the peak wavelength of Nile red red-shifted beyond the $655 \mathrm{~nm}$ expected for pure ethylene glycol because of water entrainment from the surrounding air. Thus, the largest shift, which occurs in $\mathrm{EtOH}$ with the smallest water content $(0.02 \%)$ can be explained by water entrainment. The shift obviously decreases when the water content in $\mathrm{EtOH}$ increases (Figure 5a) because the effect of water entrainment becomes less significant with increasing water content

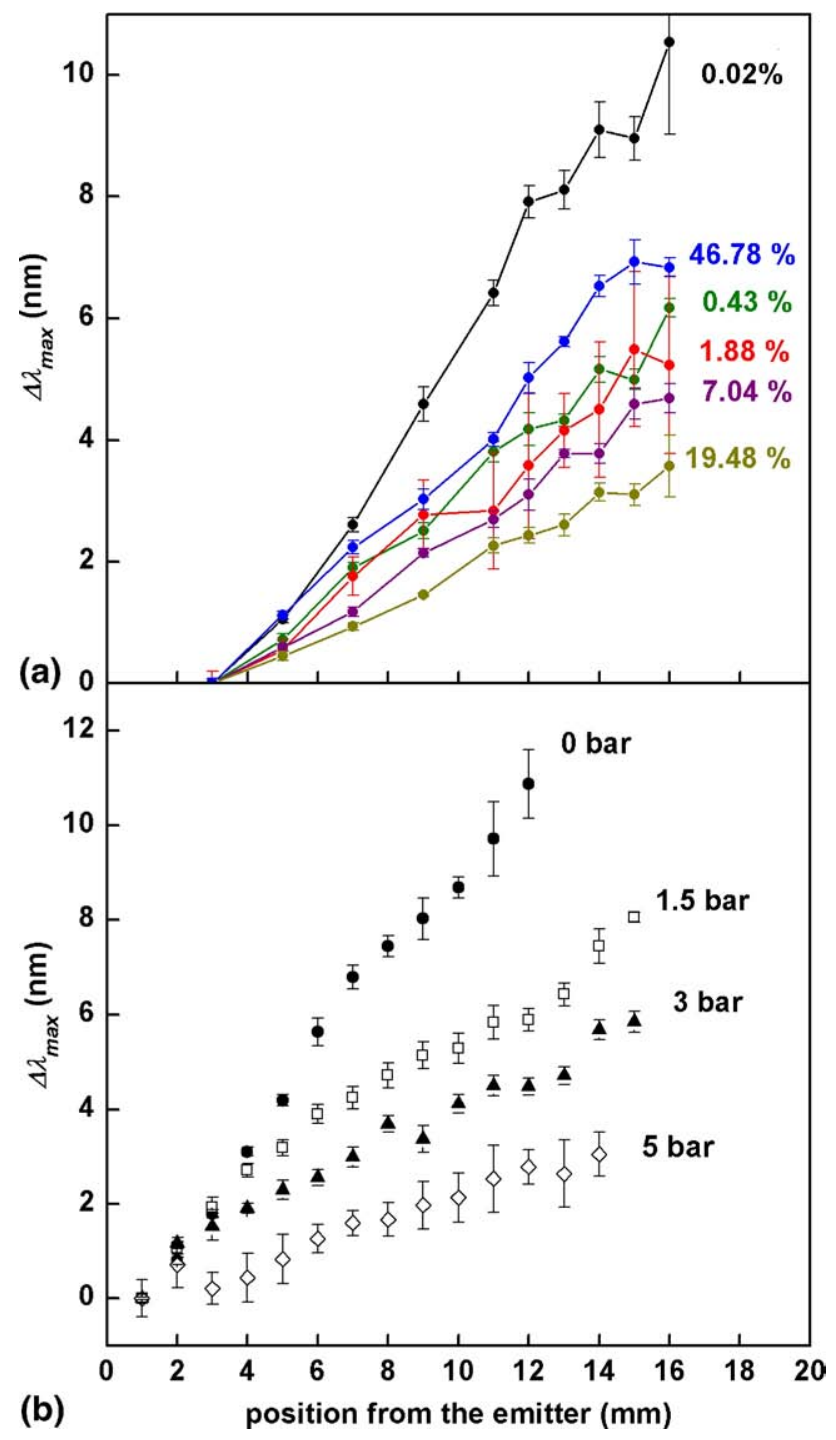

Figure 5. (a) $\Delta \lambda_{\max }$ of Nile red in EtOH with various water contents (mass percentage). $\lambda_{\text {max,bulk }}$ of Nile red with different water contents: $0.02 \%, 639.5 \mathrm{~nm} ; 0.43 \%, 638.9 \mathrm{~nm} ; 1.88 \%, 639.5 \mathrm{~nm}$; $7.04 \%, 641.8 \mathrm{~nm} ; 19.48 \%, 645.7 \mathrm{~nm} ; 46.78 \%, 655.2 \mathrm{~nm}$. (b) $\Delta \lambda_{\max }$ of Nile red in EtOH with the sheath gas of various gas pressures along the spray axis.

in the EtOH itself. Thus, both solvent evaporation and water entrainment are responsible for the changes of solvent polarity in the plume. For high-purity $\mathrm{EtOH}$, the effect of water entrainment becomes more significant than solvent evaporation. However, neither solvent evaporation nor water entrainment can explain the exceptionally high shift in EtOH with $46.78 \%$ water. Further study will be needed for understanding this behavior.

Finally, several experimental parameters that should affect the change of the solvent polarity in the plume were studied. The influence of a sheath gas (dry $\mathrm{N}_{2}$ ) with various gas pressures was investigated. The peak wavelength shifts of Nile red along the axis of the plume are shown in Figure 5b. Water entrainment from the surrounding air can be suppressed or at least 
reduced with dry sheath gas surrounding the plume. Thus the peak wavelength shift of Nile red should be less compared with a situation without sheath gas. This is exactly what was observed (Figure 5b). However, the peak wavelength shift was still more than $2 \mathrm{~nm}$, even at the highest sheath gas pressure used (5 bar), showing that an effect other than pick-up of moisture contributes to the change in solvent polarity. Neither aggregation nor a temperature decrease during the droplet shrinking process can cause a shift of more than $2 \mathrm{~nm}$. Thus, preferential evaporation of $\mathrm{EtOH}$ is the most reasonable cause for this residual wavelength shift. Water impurities in EtOH will, in this case, influence the spectral behavior of Nile red in the electrospray plume.

Solvent volatility impacts the solvent evaporation directly, i.e., should affect the solvent polarity change in the plume. The peak wavelength shift of Nile red in ACN, another common solvent used in ESI-MS, which evaporates even faster than $\mathrm{EtOH}$ because of its higher vapor pressure, was investigated along the spray direction. The results showed that the peak wavelength of Nile red shifts further in ACN than in EtOH: for example, at $14 \mathrm{~mm}$ from the emitter (on-axis), the $\Delta \lambda$ is $12 \mathrm{~nm}$ for ACN compared with only around $9 \mathrm{~nm}$ for $\mathrm{EtOH}$, demonstrating that the solvent volatility affects the change in solvent polarity.

The influence of the high voltage applied to the capillary was investigated, too. A voltage range from 3.15 to $3.55 \mathrm{kV}$ was chosen because the spray can maintain a stable mode in this range. The peak wavelength showed no obvious shift as a function of spray voltage. Switching the polarity of the voltage did not affect the shift, either. These results demonstrate that neither the quantity nor the sign of the charges in the ESI droplets affect the evolution of the solvent polarity. The effect of the charge density during droplet shrinking can be addressed using an electrodynamic balance [50]. Preliminary results showed a minor peak wavelength shift $(<2 \mathrm{~nm}$ for a size decease of $30 \mu \mathrm{m}$ to $\approx 2$ $\mu \mathrm{m}$; data not shown), suggesting that the increase in charge density in one droplet does not influence the solvent polarity much. These measurements will be described fully in a forthcoming publication.

Finally, in ESI-MS measurements, electrolytes are usually added into the solution to facilitate ionization of the analytes. Thus, the effect of electrolytes on the solvent polarity was studied. We investigated the system with acetic acid and sodium fluoride. The trends of the shift in pure $\mathrm{EtOH}$ and those in $\mathrm{EtOH}$ with both acetic acid and sodium fluoride are quite close. These observations suggest that electrolytes do not appreciably affect the solvent polarity in the electrospray plume. It can be concluded that the addition of small amounts of electrolytes neither influences solvent evaporation nor water entrainment, the two important mechanisms responsible for the change in solvent polarity in the ESI plume.

\section{Conclusions}

In this work, the solvent polarity in the electrospray plume using solvent EtOH was studied in both the spray and radial directions. Two solvatochromic dyes, Nile red and DCM, were used as probes to investigate the solvent polarity change by an in-situ method, laserinduced fluorescence spectroscopy. The peak wavelength of Nile red exhibited an 8-12 nm red shift, and its quantum yield decreased by more than $50 \%$ over a distance of $15 \mathrm{~mm}$ in the axial direction, away from the emitter. The peak wavelength of DCM also had an 8-12 $\mathrm{nm}$ red shift, but its quantum yield increased in the axial direction. Similar phenomena were observed in the radial direction of the plume, too. Effects of temperature and concentration were found to be negligible. All these findings demonstrate that the solvent polarity directly causes the observed changes of the fluorescence emission, and the polarity increases when the droplets become smaller at the periphery of the plume or further away from the emitter.

The possible mechanisms of the solvent polarity change were discussed. Both solvent evaporation and water entrainment from surrounding air were found to contribute to the polarity change in the plume. When EtOH with very low water content was employed, the effect of water entrainment became more significant than solvent evaporation. The solvent volatility also had a significant effect on the evolution of the solvent polarity, but neither the addition of electrolytes to the solution nor the ESI spray voltage influenced the solvent polarity change.

\section{Acknowledgments}

The authors thank Pavel Sagulenko and Dr. Weihua Zhang for help at several stages of this project, and Dr. Ulrich Krieger for performing the electrodynamic balance experiment. The authors acknowledge support for this research by the Swiss National Science Foundation, grant no. 200020-124663.

\section{References}

1. Nemes, P.; Marginean, I.; Vertes, A. Spraying Mode Effect on Droplet Formation and Ion Chemistry in Electrosprays. Anal. Chem. 2007, 79, 3105-3116.

2. Marginean, I.; Kelly, R. T.; Prior, D. C.; LaMarche, B. L.; Tang, K. Q.; Smith, R. D. Analytical Characterization of the Electrospray Ion Source in the Nanoflow Regime. Anal. Chem. 2008, 80, 6573-6579.

3. Enke, C. G. A Predictive Model for Matrix and Analyte Effects in Electrospray Ionization of Singly-Charged Ionic Analytes. Anal. Chem. 1997, 69, 4885-4893.

4. Zhou, S.; Cook, K. D. A Mechanistic Study of Electrospray Mass Spectrometry: Charge Gradients within Electrospray Droplets and Their Influence on Ion Response. J. Am. Soc. Mass Spectrom. 2001, 12, 206-214.

5. Boekman, C. F.; Bylund, D.; Markides, K. E.; Sjoberg, J. R. Relating Chromatographic Retention and Electrophoretic Mobility to the Ion Distribution Within Electrosprayed Droplets. J. Am. Soc. Mass Spectrom. 2006, 17, 318-324.

6. Tang, K.; Smith, R. D. Physical/Chemical Separations in the Break-Up of Highly Charged Droplets from Electrosprays. J. Am. Soc. Mass Spectrom. 2001, 12, 343-347.

7. Duft, D.; Achtzehn, T.; Mueller, R.; Huber, B. A.; Leisner, T. Coulomb Fission-Rayleigh Jets from Levitated Microdroplets. Nature 2003, 421, 128.

8. Cole, R. B. Some Tenets Pertaining to Electrospray Ionization Mass Spectrometry. J. Mass Spectrom. 2000, 35, 763-772. 
9. Olumee, Z.; Callahan, J. H.; Vertes, A. Droplet Dynamics Changes in Electrostatic Sprays of Methanol-Water Mixtures. J. Phys. Chem. A 1998, 102, 9154-9160.

10. Tang, K.; Gomez, A. On the Structure of an Electrostatic Spray of Monodisperse Droplets. Phys. Fluids 1994, 6, 2317-2332.

11. Smith, J. N.; Flagan, R. C.; Beauchamp, J. L. Droplet Evaporation and Discharge Dynamics in Electrospray Ionization. J. Phys. Chem. A 2002, 106, 9957-9967.

12. Wortmann, A.; Kistler-Momotova, A.; Zenobi, R.; Heine, M. C.; Wilhelm, O.; Pratsinis, S. E. Shrinking Droplets in Electrospray Ionization and Their Influence on Chemical Equilibria. J. Am. Soc. Mass Spectrom. 2007, 18, 385-393.

13. Wang, H.; Agnes, G. R. Kinetically Labile Equilibrium Shifts Induced by the Electrospray Process. Anal. Chem. 1999, 71, 4166-4172.

14. Wang, H.; Agnes, G. R. Evaluation of Electrospray Mass Spectrometry as a Technique for Quantitative Analysis of Kinetically Labile Solution Species. Anal. Chem. 1999, 71, 3785-3792.

15. Dole, M.; Mack, L. L.; Hines, R. L.; Mobley, R. C.; Ferguson, L. D.; Alice, M. B. Molecular Beams of Macroions. J. Chem. Phys. 1968, 49, 2240-2249.

16. Iribarne, J. V.; Thomson, B. A. On the Evaporation of Small Ions from Charged Droplets. J. Chem. Phys. 1976, 64, 2287-2294.

17. Kebarle, P. A Brief Overview of the Present Status of the Mechanisms Involved in Electrospray Mass Spectrometry. J. Mass Spectrom. 2000, 35, 804-817.

18. Kebarle, P.; Tang, L. From Ions in Solution to Ions in the Gas Phase-the Mechanism of Electrospray Mass-Spectrometry. Anal. Chem. 1993, 65, A972-A986.

19. Nguyen, S.; Fenn, J. B. Gas-Phase Ions of Solute Species from Charged Droplets of Solutions. Proc. Natl. Acad. Sci. U.S.A. 2007, 104, 1111-1117.

20. Tang, L.; Kebarle, P. Dependence of Ion Intensity in Electrospray Mass-Spectrometry on the Concentration of the Analytes in the Electrosprayed Solution. Anal. Chem. 1993, 65, 3654-3668.

21. Illuminati, G. Solvent Effects on Selected Organic and Organometallic Reactions. Guidelines to Synthetic Applications. In Techniques of Chemistry; Dack, M. R. J., Ed.; Chap XII. Series: Solutions and Solubilities Part II; J. Wiley and Sons: London, 1976; p. 159.

22. Engberts, J. B. F. N. Mixed Aqueous Solvent Effects on Kinetics and Mechanisms of Organic Reactions. In Water: A Comprehensive Treatise; Franks, F., Ed.; Vol. VI; Chpap IV; Series: Recent Advances; Plenum Press: New York, 1979; p. 139.

23. Reichardt, C. Solvents and Solvent Effects in Organic Chemistry; 3rd ed.; Wiley-VCH: Weinhem, 2003; p. 93.

24. Sekiguchi, Y.; Takayama, S.; Gotanda, T.; Sano, K. Importance of Solvent Polarity in the Equilibrium Reaction of Leuco Dye and Developer. Chem. Lett. 2006, 35, 458-459.

25. Fabris, D. Mass Spectrometric Approaches for the Investigation of Dynamic Processes in Condensed Phase. Mass Spectrom. Rev. 2005, 24, $30-54$.

26. Marquez, C. A.; Wang, H. Y.; Fabbretti, F.; Metzger, J. O. ElectronTransfer-Catalyzed Dimerization of Trans-Anethole: Detection of the Distonic Tetramethylene Radical Cation Intermediate by Extractive Electrospray Ionization Mass Spectrometry. J. Am. Chem. Soc. 2008, 130, 17208-17209.

27. Zhu, L.; Gamez, G.; Chen, H. W.; Huang, H. X.; Chingin, K.; Zenobi, R. Real-Time, On-Line Monitoring of Organic Chemical Reactions Using Extractive Electrospray Ionization Tandem Mass Spectrometry. Rapid Commun. Mass Spectrom. 2008, 22, 2993-2998.

28. Cole, R. B.; Harrata, A. K. Solvent Effect on Analyte Charge-State, Signal Intensity, and Stability in Negative-Ion Electrospray Mass-SpectrometryImplications for the Mechanism of Negative-Ion Formation. J. Am. Soc. Mass Spectrom. 1993, 4, 546-556.

29. Wang, G.; Cole, R. B. Effects of Solvent and Counterion on Ion Pairing and Observed Charge States of Diquaternary Ammonium Salts in Electrospray Ionization Mass Spectrometry. J. Am. Soc. Mass Spectrom. 1996, 7, 1050-1058.
30. Lampa-Pastirk, S.; Beck, W. F. Intramolecular Vibrational Preparation of the Unfolding Transition State of ZnII-Substituted Cytochrome C. J. Phys. Chem. B 2006, 110, 22971-22974.

31. Gupta, M. N.; Batra, R.; Tyagi, R.; Sharma, A. Polarity Index: The Guiding Solvent Parameter for Enzyme Stability in Aqueous-Organic Cosolvent Mixtures. Biotechnol. Prog. 1997, 13, 284-288.

32. Griffith, O. H.; Dehlinger, P. J.; Van, S. P. Shape of Hydrophobic Barrier of Phospholipid Bilayers (Evidence for Water Penetration in BiologicalMembranes). J. Membr. Biol. 1974, 15, 159-192.

33. Zhou, S.; Cook, K. D. Probing Solvent Fractionation in Electrospray Droplets with Laser-Induced Fluorescence of a Solvatochromic Dye. Anal. Chem. 2000, 72, 963-969.

34. Mataga, N.; Kaifu, Y.; Koizumi, M. Solvent Effects Upon Fluorescence Spectra and the Dipole Moments of Excited Molecules. Bull. Chem. Soc. Jpn. 1956, 29, 465-470.

35. Lippert, V. Z. Dipole Moment and Electronic Structure of Excited Molecules. Z. Naturforsche 1957, 10a, 541-545.

36. Kosower, E. M. The Effect of Solvent on Spectra I. A New Empirical Measure of Solvent Polarity-Z-Values. J. Am. Chem. Soc. 1958, 80, 3253-3260.

37. Sarkar, N.; Das, K.; Nath, D. N.; Bhattacharyya, K. Twisted ChargeTransfer Process of Nile Red in Homogeneous Solutions and in Faujasite Zeolite. Langmuir 1994, 10, 326-329.

38. Bondarev, S. L.; Knyukshto, V. N.; Stepuro, V. I.; Stupak, A. P.; Turban, A. A. Fluorescence and Electronic Structure of the Laser Dye DCM in Solutions and in Polymethylmethacrylate. J. Appl. Spectrosc. 2004, 71, 194-201.

39. Lin, C. T.; Mahloudji, A. M.; Li, L.; Hsiao, M. W. Molecular Aggregation of Rhodamine $6 \mathrm{~g}$ Probed by Optical and Electrochemical Techniques. Chem. Phys. Lett. 1992, 193, 8-16.

40. Peng, X.; Draney, D. R.; Volcheck, W. M.; Bashford, G. R.; Lamb, D. T.; Grone, D. L.; Zhang, Y.; Johnson, C. M. Phthalocyanine Dye as an Extremely Photostable and Highly Fluorescent Near-Infrared Labeling Reagent. Proceedings of the Conference on Optical Molecular Probes for Biomedical Applications; Achilefu, S., Bornhop, D. J., Raghavachari, R., Eds.; Spie-Int Soc Optical Engineering, San Jose, CA, 2006.

41. Magde, D.; Rojas, G. E.; Seybold, P. G. Solvent Dependence of the Fluorescence Lifetimes of Xanthene Dyes. Photochem. Photobiol. 1999, 70, 737-744.

42. Magde, D.; Wong, R.; Seybold, P. G. Fluorescence Quantum Yields and Their Relation to Lifetimes of Rhodamine $6 \mathrm{~g}$ and Fluorescein in Nine Solvents: Improved Absolute Standards for Quantum Yields. Photochem. Photobiol. 2002, 75, 327-334.

43. Lakowicz, J. R. Principles of Fluorescence Spectroscopy; 3rd ed.; Springer: Berlin, 2006; pp 206-209.

44. Selwyn, J. E.; Steinfeld, J. I. Aggregation Equilibria of Xanthene Dyes. J. Phys. Chem. 1972, 76, 762-774.

45. Basting, D.; Ouw, D.; Schafer, F. P. The Phenoxazones: A New Class of Laser Dyes. Opt. Commun. 1976, 18, 260-262.

46. Satpati, A.; Senthilkumar, S.; Kumbhakar, M.; Nath, S.; Maity, D. K.; Pal, $\mathrm{H}$. Investigations of the Solvent Polarity Effect on the Photophysical Properties of Coumarin-7 Dye. Photochem. Photobiol. 2005, 81, 270-278.

47. Pemberton, R. C.: Mash, C. J. Thermodynamic Properties of Aqueous Non-Electrolyte Mixtures, I. I. Vapor Pressures and Excess Gibbs Energies for Water + Ethanol at 303.15 to $363.15 \mathrm{~K}$ Determined by an Accurate Static Method. J. Chem. Thermodynamics 1978, 10, 867-888.

48. Innocenzi, P.; Malfatti, L.; Costacurta, S.; Kidchob, T.; Piccinini, M.; Marcelli, A. Evaporation of Ethanol and Ethanol-Water Mixtures Studied by Time-Resolved Infrared Spectroscopy. J. Phys. Chem. A 2008, 112, 6512-6516.

49. Katta, V.; Chait, B. T. Hydrogen/Deuterium Exchange Electrospray Ionization Mass Spectrometry: A Method for Probing Protein Conformational Changes in Solution. J. Am. Chem. Soc. 1993, 115, 6317-6321.

50. Samuel, F. W.; Agnes, G. R. Study of Chemistry in Droplets with Net Charge Before and After Coulomb Explosion: Ion-Induced Nucleation in Solution and Implications for Ion Production in an Electrospray. Anal. Chem. 2005, 77, 3189-3197. 